\section{Urate Transporters: Transforming the Face of Hyperuricemia and Gout}

...the causes which predispose to gout... are either such as produce an increased formation of uric acid in the system, or which lead to its retention in the blood. - A.B. Garrod, $1863^{1}$

Although some readers of The Journal may find the September article by Torres, et $a l^{2}$ a challenge, we believe the effort to persevere will be well worth it. This is because the report nicely illustrates how the burgeoning field of analysis of common variants in gene sequences (polymorphisms), when combined with scrupulous attention to the clinical characteristics of affected patients, can simplify and extend understanding of complicated disease mechanisms and, potentially, contribute to more informed clinical evaluation and management decisions. The disease at issue here is gout, an inflammatory metabolic disorder afflicting over 8 million of the estimated 40 million hyperuricemic American adults.

Before discussing the Torres, et al report $^{2}$, we present an historical overview of the pathogenesis of hyperuricemia and gout, with the aim of providing a context for work described therein.

That hyperuricemia is a necessary, but not sufficient, risk factor for gout was postulated in 1859 and 1863 by A.B. Garrod $^{1}$, who also proposed that crystals of "urate of soda" deposited in tissues were the cause of gouty inflammation and could remain for protracted periods, causing local destruction (tophaceous gout). An additional remarkable insight was that "...the kidneys are implicated in gout, probably in its early, but certainly in its chronic stages, and the renal affection, perhaps only functional at first, subsequently becomes structural...." As affirmed above, Garrod also clearly recognized the distinction between gout arising from uric acid overproduction and from impaired uric acid elimination.

Garrod's exposition of the hyperuricemia, urate crystal deposition, and acute and chronic inflammation pathogenetic triad in gout was verified and extended over the next
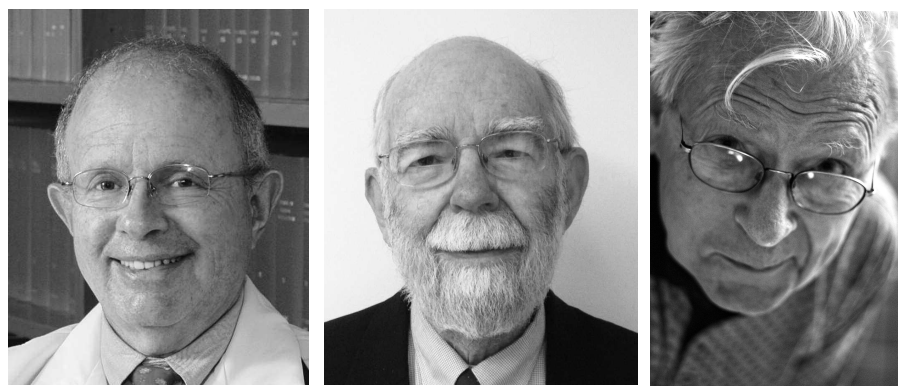

century, particularly in the 1950s and 1960s. In these decades, biochemical, physiological, pharmacological, and isotopic labeling methods of metabolic analysis were applied to study the details of uric acid production and disposal in normal persons and persons with gout. Among the results of these investigations were delineation of the pathways of purine nucleotide synthesis and of nucleotide degradation to uric acid, as well as quantitation of the size and dynamics of the body's urate pool and of the contributions of renal and gastrointestinal sites to urate elimination from the body ${ }^{3,4}$. This work confirmed that hyperuricemia in humans resulted from either excessive rates of synthesis or from impaired renal elimination of uric acid; moreover, in conjunction with clinical studies, it provided the framework for rational urate-lowering pharmacotherapies (uricosuric agents and xanthine oxidase inhibitors) for gout.

Over the ensuing decades, additional clinical, epidemiological, and experimental studies identified an extended array of acquired influences on uric acid balance ${ }^{5,6,7}$ (Figure 1). Moreover, they called attention to the likelihood that in hyperuricemic/gouty individuals, as in everyone else, multiple influences determine the serum urate concentrations and that both overproduction and impaired disposal of uric acid may coexist.

A constitutional (genetic) risk for gout was implicit in the aphorisms of Hippocrates (5th century BCE), and in observations in the first and second centuries $\mathrm{CE}$ regarding a "familial gouty diathesis". No significant genetic insights, however, followed until the 20th century, when A.E. Garrod included gout among the "inborn errors of metabolism." Studies of families and in ethnic groups with high gout prevalence also suggested several modes of hereditary transmission.

In the past 50 years, rare monogenic defects leading to gout with either accelerated purine nucleotide and uric acid synthesis [e.g., hypoxanthine guanine phosphoribosyltransferase (HPRT) deficiencies with Lesch-Nyhan disease and variants; phosphoribosylpyrophosphate synthetase (PRPPS)

See Gene polymorphisms differentiate patients with gout, September Issue, page 1863

Personal non-commercial use only. The Journal of Rheumatology Copyright @ 2014 . All rights reserved. 


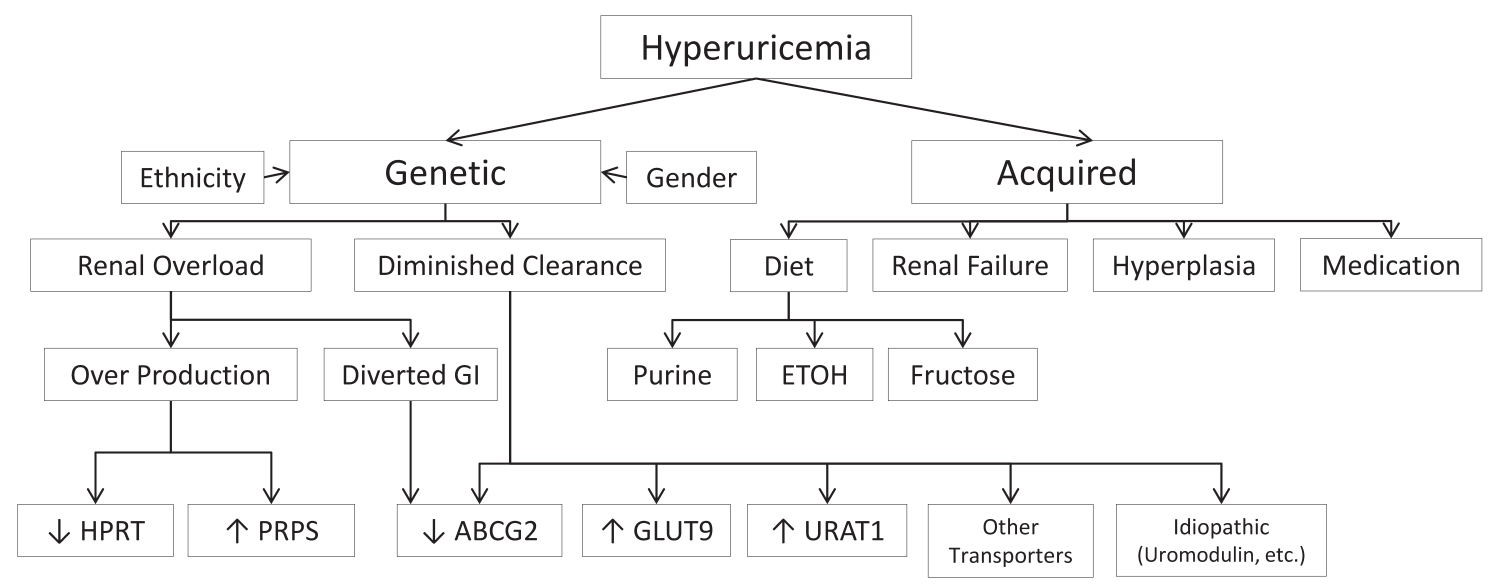

Figure 1. Factors in hyperuricemia in 2014.

overactivity] or impaired renal uric acid excretion (e.g., mutations in uromodulin or renin) have strengthened the concept that purine nucleotide and uric acid overproduction and impaired renal uric acid clearance comprise the mechanistic bases of hyperuricemia ${ }^{9}$. Study of these inborn errors also established that the actions of multiple genes expressed in distinctive physiological and biochemical pathways were involved in the pathogenesis of hyperuricemia and gout.

Nevertheless, until recently, 2 problems impeded further analysis of genetically determined risk in gout. First, the monogenic defects associated with gout are indeed rare. For example, despite extensive screening efforts, mutations in HPRT or PRPPS (PRPS1) genes associated with purine nucleotide and urinary uric acid overproduction are found in substantially fewer than $1 \%$ of all patients with gout and in no more than perhaps $3 \%$ of patients with urinary uric acid overexcretion. Second, the potential genetic contributions to gout risk in the $85 \%$ to $90 \%$ of patients with gout with impaired renal uric acid clearance ${ }^{2,4,9}$ remained unclear until molecular analyses of urate transporters in the kidney and other organs became accessible. In recent years, cloning, sequencing, and tissue/organ expression profiles of multiple renal anion transporter/exchanger genes have been accomplished ${ }^{9}$, revealing an array of carrier molecules involved in the renal proximal tubular urate reabsorption and secretion functions operationally defined in prior years by classical renal physiologic and pharmacologic analyses ${ }^{10}$.

Among the many important features of urate transporters identified to date, 3 warrant comment in the context of their role in the genetics of gout and their pertinence to the article under discussion. First, some urate transporters, notably the secretory transporter BCRP (encoded by the $A B C G 2$ gene), are expressed in the gut as well as in the kidney and play a role in active intestinal elimination of urate $e^{9,11,12}$. Second, genes encoding both net reabsorptive and net secretory urate transporters are polymorphic, showing DNA sequence variations that are common, some of which are also identifiable as risk (or protective) alleles with respect to their higher (or lower) frequency of appearance in hyperuricemic persons/patients with gout than in corresponding persons without gout ${ }^{9,11,13}$. Third, the ranges of endogenous and drug metabolites other than urate that are substrates for the various transporters are broad, implying, for example, that the fructose and glucose (as well as urate) specificities of the reabsorptive transporter GLUT9 could be key determinants of a hyperuricemia/gout risk imparted by ingestion of high levels of these sugars among persons carrying a risk allele for the corresponding $S L C 2 A 9$ gene ${ }^{9,13}$.

The study by Torres, et $a l^{2}$, as well as the research of others directly ${ }^{11,12}$ or indirectly ${ }^{14,15}$, address both of the progress-limiting issues identified above. Distinguishing methodological features of the Torres, et $a l^{2}$ study include unusually careful control of diet, medications, and sample accession, permitting accurate classification of the daily urinary uric acid excretion status of 104 ambulatory Spanish patients with primary gout (i.e., gout unrelated to a known enzymopathy or secondary to another medical condition); comparison of the patients with gout versus normal Spanish population controls with regard to the frequencies of 5 single nucleotide polymorphisms (SNP) associated, in other population groups, with urate transporter alleles altering the risk for hyperuricemia and gout; and creation of a risk index to assess possible interactions among risk alleles for the urate transporters URAT1 (encoded by gene SLC22A12), GLUT9, and BCRP. (It is important to note that the urinary uric acid excretor classification scheme ${ }^{16}$ utilized in this study $^{2}$ distinguishes uric acid underexcretors from normoexcretors, but includes in the latter group patients who, on the basis of uncorrected daily uric acid excretion, have been described in prior literature as uric acid overexcretors.)

The key findings of the SNP analysis described here ${ }^{2}$ newly establish and/or further extend the results of studies in a number of other population groups. First, risk alleles for the reabsorptive transporters URAT1 and GLUT9 were in this study significantly and uniquely associated with uric acid underexcretion, but never with normoexcretion.

Personal non-commercial use only. The Journal of Rheumatology Copyright (c) 2014. All rights reserved. 
Second, and in contrast, the risk allele for BCRP was positively associated with both underexcretion and normoexcretion, but the association was much stronger with normoexcretion. The importance of the latter observation is that it provides confirmation of work reported from Japan ${ }^{11,12}$, implicating the polymorphism in the $A B C G 2$ gene as a risk allele for hyperuricemia/gout by a previously undescribed mechanism: reduced intestinal urate disposal, thus placing a greater burden on the kidneys to assure uric acid balance. The consequence is hyperuricemia and an increased risk for gout, despite urinary excretion of high normal or even increased amounts of uric acid. This newly recognized mechanism for hyperuricemia is supported by studies in abcg2-knockout mice in which both serum and urinary uric acid excretion are increased and gut excretion is decreased compared with control mice ${ }^{12}$. It is also in accord with earlier evaluations of uric acid excretion in gouty and normal control subjects, each of whom were studied over a broad range of experimentally manipulated serum urate concentrations ${ }^{14,15}$. There, gouty quantitative overexcretors shared the same qualitative defect found in patients with gout who excreted normal quantities of urinary uric acid at baseline. In hindsight, these observations fit well with defective urate secretion into both the gut and the kidney. On the basis of the high frequency of ABCG2 risk alleles in human populations $2,9,11$, it seems likely that "uric acid overexcretion due to extrarenal underexcretion"11 is much more common than overexcretion resulting from monogenic disorders with purine nucleotide and uric acid overproduction.

Finally, the additive or synergistic effect of multiple common risk alleles (for the transporters URAT1, GLUT9, and BCRP) on the gout risk index ${ }^{2}$ likely reflects interactions among risk alleles that may ultimately provide the background for much (or even most) gout with diminished uric acid renal clearance. This hypothesis is made particularly attractive in light of the fact that substrates for some urate transporter systems (e.g., GLUT9) include dietary or other components known to promote the risk for hyperuricemia and gout $^{5,7,9}$.

MICHAEL A. BECKER, MD;

Professor Emeritus,

The University of Chicago Pritzker School of Medicine, Chicago, IL;

PETER A. SIMKIN, MD,

Professor Emeritus of Medicine,

University of Washington School of Medicine,

Seattle, WA;

LEIF B. SORENSEN, $M D, P h D$

Professor Emeritus,

The University of Chicago Pritzker School of Medicine, Chicago, IL, USA.

Address correspondence to Dr. Becker, 237 East Delaware Place, Chicago, IL 60637-1713, USA. E-mail: becker@medicine.bsd.uchicago.edu

\section{REFERENCES}

1. Garrod AB. The nature and treatment of gout and rheumatic gout 2nd ed. London: Walton and Maberly; 1863.

2. Torres R, de Miguel E, Bailen R, Banegas J, Puig J. Tubular urate transporter gene polymorphisms discriminate patients with gout who have normal and decreased urinary uric acid excretion. J Rheumatol 2014;41:1863-70.

3. Wyngaarden JB, Kelley WN. Gout and hyperuricemia. New York: Grune and Stratton; 1976.

4. Sorensen LB. Role of the intestinal tract in the elimination of uric acid. Arthritis Rheum 1965;8:694-706.

5. Choi HK, Atkinson K, Karlson EW, Willett W, Curhan G. Purine-rich foods, dairy and protein intake, and the risk of gout in men. N Engl J Med 2004;350:1093-103.

6. Choi HK, Atkinson K, Karlson EW, Curhan G. Obesity, weight change, hypertension, diuretic use, and risk of gout in men. Arch Intern Med 2005;165:742-8.

7. Choi HK, Willett W, Curhan G. Fructose-rich beverages and risk of gout in women. JAMA 2010;304:2270-8

8. Garrod AE. The inborn factors in disease: an essay. London and New York: Oxford University Press (Clarendon); 1931.

9. Dalbeth N, Merriman T. Hyperuricemia and gout. In: Valle D, Beaudet AL, Vogelstein B, Kinzler KW, Antonarakis SE, Ballabio A, et al, editors. The online metabolic and molecular bases of inherited disease. New York: McGraw-Hill; 2012; Chapter 106.

10. Rieselbach RE. Renal handling of uric acid. Adv Exp Med Biol 1976;76B;1-22.

11. Matsuo H, Takada T, Nakayama A, Shimizu T, Sakayama M, Shimizu S, et al. ABCG2 dysfunction increases the risk of overload hyperuricemia. Nucleosides Nucleotides Nucleic Acids 2014:33:266-74

12. Takada T, Ichida K, Matsuo H, Nakayama A, Murakami K, Yamanashi $\mathrm{Y}$, et al. ABCG2 dysfunction increases serum uric acid by decreased intestinal urate excretion. Nucleosides Nucleotides Nucleic Acids 2014;33:275-81.

13. Merriman TR, Choi HK, Dalbeth N. The genetic basis of gout. Rheum Dis Clin North Am 2014;40:279-90.

14. Simkin PA. Uric acid excretion in patients with gout [letter]. Arthritis Rheum 1979;22:98-9.

15. Fontenaille C, Guiheniuc P, Heron B, Prost A, Lucas J. Renal excretion of urate in normal and gouty subjects at high, normal, and low plasma urate levels. Biomedicine 1974;21:382-5.

16. Puig JG, Torres RJ, de Miguel E, Sanchez A, Bailen R, Banegas JR. Uric acid excretion in healthy subjects: a nomogram to assess the mechanisms underlying purine metabolic disorders. Metabolism 2012;61:512-8.

J Rheumatol 2014;41:1910-2; doi:10.3899/jrheum.141019

Personal non-commercial use only. The Journal of Rheumatology Copyright @ 2014 . All rights reserved. 